\title{
LITERARY THEORY AND THE SCIENCES
}

Guest Editor

Călin-Andrei Mihăilescu 